\title{
Investigation of the electric drive system of the lifting unit with parallel coordinate correction
}

\author{
Sergey Reshetnyak ${ }^{1,2}$, Yuriy Maksimenko ${ }^{2}$, and Alla Zakharova $^{3 *}$ \\ ${ }^{1}$ IPRON RAS, 111020, Moscow, Russia \\ ${ }^{2}$ MISIS, 119991, Moscow, Russia \\ ${ }^{3}$ T.F. Gorbachev Kuzbass State Technical University, 650000, Kemerovo, Russia
}

\begin{abstract}
In the presented publication, the issues of improving the energy efficiency of the electric drive system, in relation to mine lifting installations, are considered. A block diagram of the electric drive system of a mine lifting installation with parallel coordinate correction is considered, as well as a mathematical description of the presented system. It should be noted that the conducted research will significantly affect the improvement of the level of safety and accident-free operation of the mine lifting plant, which will increase the competitiveness of coal mining by underground method.
\end{abstract}

\section{Introduction}

The development of the Russian mining industry requires modern transport schemes for the extraction of rock mass $[1-3,11,12]$. The leading role as a transport equipment for the extraction of rock mass during underground mining operations is played by a lifting unit. The operation of the entire mining enterprise depends on the reliable and uninterrupted operation of the lifting plant. Therefore, improving the operational properties of the lifting plant is an urgent task.

Most modern lifting equipment is equipped with an electric drive system TP-D (thyristor converter-motor). This is due to a few advantages of a DC motor in relation to AC motors. Scientists: V.E. Katolikov, A.D. Dinkel, V.S. Tulin, A.M. Sedunin and others were engaged in the development and research of mine lifting installations using the TP-D system.

One of the ways to increase the operational properties is to increase the service life of the elastic elements (ropes) of the lifting system. The presented method is carried out by limiting the dynamic loads by using the damping properties of the electric drive $[4,5]$. During the operation of the lifting system, various types of vibrations occur in the elastic elements: longitudinal, transverse, torsional. These fluctuations significantly affect the wear of the ropes in the lifting system. Accordingly, it is necessary to create an EMC (electromechanical system) with the possibility of damping vibrations in elastic elements by regulating (correcting) feedback signals in the electric drive system of the lifting unit [5].

\footnotetext{
* Corresponding author: zaharovaag@kuzstu.ru
} 


\section{Results and discussion}

The closed system of the three-mass EMC lifting system with parallel coordinate correction is shown in Fig. 1. In the presented scheme, the following feedbacks are present: rigid and flexible in speed, rigid and flexible in current, rigid and flexible in elastic load moment.

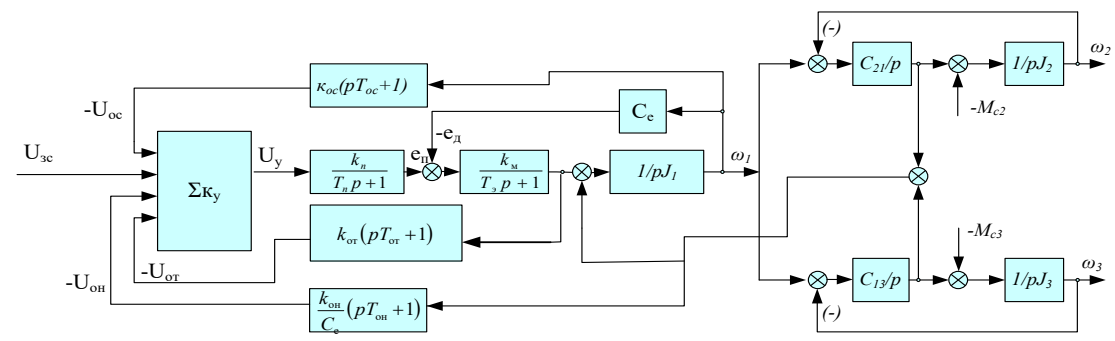

Fig. 1. Block diagram of the EMC of the lifting unit with parallel coordinate correction.

The use of the method of normalized transfer functions [2,3] made it possible to create a mathematical model of the EMC of the lifting unit with parallel coordinate correction:

$$
\begin{aligned}
& \frac{\kappa_{2} \kappa_{3}}{m} \tau_{\mathrm{M}} \tau_{1}^{4}+\frac{\kappa_{2} \kappa_{3}}{m} \tau_{\mathrm{T}} \tau_{\mathrm{M}} \tau_{1}^{4}=\tau^{6} \\
& \frac{\kappa_{2} \kappa_{3}}{m}\left(1+\lambda_{2}\right) \tau_{\mathrm{m}} \tau_{1}^{4}+\frac{\kappa_{2} \kappa_{3}}{n} \tau_{\mathrm{c}} \tau_{1}^{4}=6 \xi \tau^{5} \\
& \frac{\kappa_{2} \kappa_{3}}{n}\left(1+\lambda_{1}\right) \tau_{1}^{4}+\left(\frac{\kappa_{2} \kappa_{3}(1+n)+\kappa_{2} n+\kappa_{3}}{\gamma n}\right)\left(1+\tau_{\text {т }}\right) \tau_{\mathrm{M}} \tau_{1}^{2}+ \\
& +\left(\frac{\kappa_{2} \kappa_{3}(1+n)}{\gamma n}\right) \tau_{\mathrm{H}} \tau_{\mathrm{M}} \tau_{1}^{2}=3 \tau^{4}+12 \xi^{2} \tau^{4} \\
& \left(\frac{\kappa_{2} \kappa_{3}(1+n)+\kappa_{2} n+\kappa_{3}}{\gamma n}\right)\left(1+\lambda_{2}\right) \tau_{\mathrm{M}} \tau_{1}^{2}+\lambda_{3}\left(\frac{\kappa_{2} \kappa_{3}(1+n)}{\gamma n}\right) \tau_{\mathrm{M}} \tau_{1}^{2}+ \\
& +\left(\frac{\kappa_{2} n+\kappa_{3}}{n}\right) \tau_{\mathrm{c}} \tau_{1}^{2}=8 \xi^{3} \tau^{3}+12 \xi \tau^{3} \\
& \left(\frac{n \kappa_{2}+\kappa_{3}}{n}\right)\left(1+\lambda_{1}\right) \tau_{1}^{2}+\frac{1}{\gamma}\left(\kappa_{2}+\kappa_{3}\right) \tau_{\mathrm{H}} \tau_{\mathrm{M}}+\tau_{\mathrm{T}} \tau_{\mathrm{M}}+\tau_{\mathrm{M}}=3 \tau^{2}+12 \xi^{2} \tau^{2} \\
& \tau_{\mathrm{M}}\left(1+\lambda_{2}\right)+\frac{1}{\gamma} \lambda_{3}\left(\kappa_{2}+\kappa_{3}\right) \tau_{\mathrm{M}}+\tau_{\mathrm{c}}=6 \xi \tau,
\end{aligned}
$$


where: $\gamma=\left(J_{1}+J_{2}+J_{3}\right) / J_{1}=1+\kappa_{2}+\kappa_{3}$ - the relative moment of inertia of the three-mass EMC; $\kappa_{2}=J_{2} / J_{1}$ - the relative value of the moment of inertia of the second mass; $\kappa_{3}=J_{3} / J_{1}$ - the relative value of the moment of inertia of the third mass; $n=C_{13} / C_{21}$ - the relative value of the stiffness coefficient; $J_{1}$ - the reduced moment of inertia of the first mass, including the moment of inertia of the engine, gearbox, winding body, guide pulleys; $J_{2}$ - the reduced moment of inertia of the second mass, loaded lifting vessel; $J_{3}$ - the reduced moment of inertia of the third mass, empty lifting vessel; $C_{21}-$ the reduced stiffness of the rope strings between the winding body and the loaded lifting vessel; $C_{13}$-reduced stiffness of the rope strings between the winding body and the empty lifting vessel; $\xi$ - relative coefficient of vibration damping; $\tau_{\mathrm{c}}=T_{\mathrm{c}} / T_{\text {э }}$ - relative parameter of the time constant of the flexible feedback on the system speed; $\tau_{\mathrm{T}}=T_{\mathrm{T}} / T_{{ }_{3}}$ - relative parameter of the time constant of the flexible system current feedback; $\tau_{\ni}=T_{\text {н }} / T_{\text {э }}$ - the relative parameter of the time constant of the flexible feedback on the elastic moment of the system; $\tau=T / T_{\text {э }}$ - the relative time constant that characterizes the system performance; $\tau_{\mathrm{M}}=T_{\mathrm{M}} / T_{\ni}$ - relative electromechanical time constant EMC; $\tau_{1}=T_{1} / T_{\text {э }}$ - the relative time constant that characterizes the natural oscillation frequencies of the three-mass EMC; $T_{\mathrm{M}}=\left(J_{1}+J_{2}+J_{3}\right) R_{\mathrm{g}} / C_{e}{ }^{2}=\left(J_{1}+J_{2}+J_{3}\right) / \kappa_{\mathrm{M}} C_{e}$ - electromechanical time constant of the system; $T_{1}^{2}=J_{1} / C_{21}$ - the square of the time constant that characterizes the natural oscillation frequencies of the three-mass EMC, $R_{\mathrm{g}}$ - the active resistance of the armature winding of the motor; $C_{\mathrm{e}}-$ the internal feedback of the motor on the EMF [2, 3].

The most effective way to dampen vibrations in the elastic elements of the lifting system is the use of negative feedback on the elastic moment of the load (rigid and flexible). In addition, the number of feedbacks should not exceed four, due to the complexity of setting up negative feedback loops [5].

A transition process close to the aperiodic, with parallel coordinate correction, will be obtained under the condition $\xi \geq \sqrt{2} / 2$ that the following conditions must also be met: (Option $1, \tau_{\mathrm{H}} \geq 0 ; \lambda_{3} \geq 0 ; \tau_{\mathrm{T}} \geq 0 ; \lambda_{2} \geq 0$ ); (Option 2. $\tau_{\mathrm{H}} \geq 0 ; \lambda_{3} \geq 0 ; \tau_{\mathrm{T}} \geq 0$; $\left.\lambda_{1} \geq 0\right)$. In accordance with the presented variants, the regions of existence of these sets of feedbacks were obtained.

For the first variant of the set of feedbacks ( $\tau_{\mathrm{H}} \geq 0 ; \lambda_{3} \geq 0 ; \tau_{\mathrm{T}} \geq 0 ; \lambda_{2} \geq 0$ ), the domain of existence is determined by the solution of the following expression: 


$$
\begin{aligned}
& 17,995 \cdot(\gamma-1)\left(\begin{array}{l}
8,998-(x+w) \frac{0,167(15,999 \gamma-15,999-0,333 x \gamma)}{(x+w) \gamma-(x+w)-x \gamma} \\
-\frac{\gamma 2((x+w) \gamma-(x+w)-x \gamma)}{(15,999 \gamma-15,999-0,333 x \gamma)}
\end{array}\right)= \\
& =\gamma x\left(\begin{array}{l}
8,998-\frac{2,999(15,999 \gamma-15,999-0,333 x \gamma)}{(x+w) \gamma-(x+w)-x \gamma}- \\
-\frac{\gamma w}{17,995}
\end{array}\right),
\end{aligned}
$$

where: $x=\frac{\tau_{1}^{2} \kappa_{2} \kappa_{3}(1+n)}{n \gamma} ; w=\frac{\tau_{1}^{2}\left(\kappa_{2} n+\kappa_{3}\right)}{n \gamma}$, - the accepted notation characterizing the parameters of the real lifting installation.

For the second variant of the set of feedbacks $\left(\tau_{\mathrm{H}} \geq 0 ; \lambda_{3} \geq 0 ; \tau_{\mathrm{T}} \geq 0 ; \lambda_{1} \geq 0\right.$ ), the domain of existence is determined by the solution of the following expression:

$$
\begin{aligned}
& 2913,6(\gamma-1)-\frac{(863,48(\gamma-1)-17,995 x x)(\gamma+1)(x+w)}{(x+w)(\gamma+1)-\gamma x}=\frac{324,1((x+w)(\gamma+1)-\gamma x)}{47,98((\gamma-1)-\gamma x)} \gamma^{2}- \\
& -\frac{324,1((x+w)(\gamma+1)-\chi x)}{47,98((\gamma-1)-\gamma x)} \gamma+161,93 \gamma x+\frac{(863,48(\gamma-1)-17,995 \gamma)}{(x+w)(\gamma+1)-\gamma x}-\gamma^{2} w x .
\end{aligned}
$$

The resulting expressions have complex dependencies, so they are presented graphically in Fig.2 (a, b).

a)

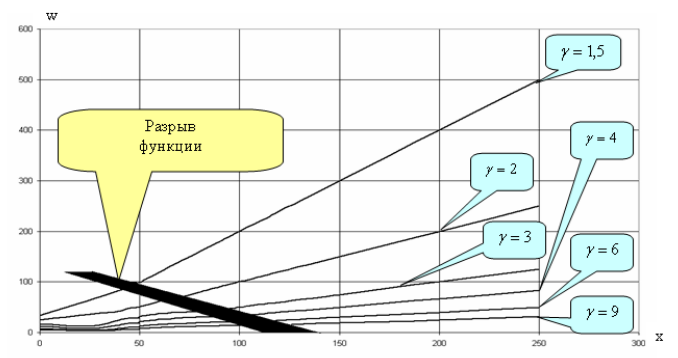

b)

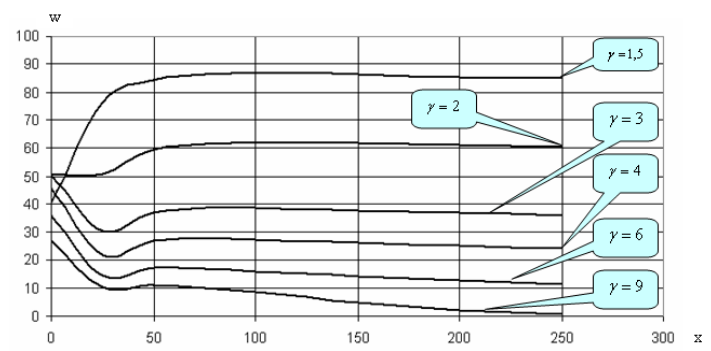

Fig. 2. Areas of existence of variants of feedbacks of the lifting system with parallel coordinate correction: a) option 1 ; b) option 2 . 


\section{Conclusion}

Research the existence of regions with a parallel coordinate's correction with a flexible negative feedback on current and elastic torque loads, and hard negative feedback on current and elastic load torque showed that, in the region of existence of the feedback there was a gap function:

when the break function is in the range $40<x<45$, when the break function is in the range $60<x<68$, when the break function is in the range $80<x<91$, when the break function is in the range of $89<x<103$, when the break function is in the range of $98<\mathrm{x}<116$, when the gap of the function lies within $102<\mathrm{x}<126$,

which introduces a restriction on the application of the presented set of feedbacks. In addition, the control structure, which contains two types of current feedbacks (rigid and flexible), compensate for each other, and do not have a noticeable effect on the dynamics of the EMC.

In the development of further research, it is proposed to build a simulation mathematical model of a lifting installation in the Matlab software product, to form a digital double for the study of various operating modes $[6,7,8,9,10]$.

Studies of the domain of existence with parallel coordinate correction with flexible negative feedbacks for current and elastic moment of load and rigid negative feedbacks for speed and elastic moment of load have shown that the use of this set of feedbacks is possible to dampen vibrations in the elastic elements of the lifting installation at all values of the relative moment of inertia of the three-mass EMC $\gamma$. This has a beneficial effect on the service life of elastic elements (ropes), thereby increasing their service life.

\section{References}

1. O. Kazanin, A. Sidorenko, A. Meshkov, S. Sidorenko, Eurasian Mining Industry, 2, $19(2020)$

2. O. Kazanin, A. Sidorenko, Y. Sirenko, ARPN Journal of Engineering and Applied Sciences, 14 (3), 732 (2019)

3. A. Sidorenko, V. Ivanov, S. Sidorenko, International Engineering and Technical Journal, 10 (1), 844 (2019)

4. S. Reshetnyak, Mining information and analytical bulletin, 10, 367 (2010)

5. A. Lyakhomsky, V. Faschilenko, S. Reshetnyak, Mining Journal, 4, 59 (2010)

6. A. Semenov, M. Semenova, Y. Bebikhov, Proceedings of the International Russian Conference on Automation (RusAutoCon 2019), 1, 19 (2019)

7. A. Semenov, I. Yakushev, A. Egorov, Modern High Technologies, 8, 56 (2017)

8. D. Kuziyev, Y. Pyatov, I. Klement'eva, D. Patronski, Mining information and analytical Bulletin, 1, 128 (2019)

9. I. Klement'eva, D. Kuziyev, Mining information-analytical bulletin, 2, 123 (2019)

10. R. Muminov, G. Raikhanova, D. Kuziev, Ugol, 5, 32 (2021)

11. V. Perekutnev, V. Zotov, Mining information and analytical bulletin, 7, 85 (2020)

12. V. Perekutnev, V. Zotov, Mining information and analytical bulletin, 6, 105 (2020) 